\title{
CARD15 gene mutations in sarcoidosis
}

\author{
M. Schürmann*, R. Valentonyte", J. Hampe ${ }^{\#}$, J. Müller-Quernheim, E. Schwinger*, S. Schreiber"
}

CARD15 gene mutations in sarcoidosis. M. Schürmann, R. Valentonyte, J. Hampe, J. Müller-Quernheim, E. Schwinger, S. Schreiber. (C)ERS Journals Ltd 2003.

ABSTRACT: Sarcoidosis, Blau syndrome and Crohn's disease are complex disorders, characterised by granulomatous inflammation affecting a variety of organs. Mutations of the CARD15 gene, on chromosome 16, have been shown to contribute significantly to Crohn's disease and to cause Blau syndrome. These factors prompted the current authors to study CARD15 mutations in sarcoidosis.

A total of 138 families were genotyped, including 302 patients with sarcoidosis and 127 patients without a family history of sarcoidosis (together with their parents), for four main coding CARD15 polymorphisms associated with increased risk of Crohn's disease. Furthermore, the gene segment that harbours Blau syndrome mutations was sequenced in 39 selected patients from 39 families with affected siblings identical for one or two parental chromosomes $16 \mathrm{~s}$ and in eight patients from multi-case families.

None of the reported Blau syndrome mutations and no new sequence alterations were found. There was an increased frequency of transmission of the rare allele of the polymorphic sites $802 \mathrm{C}>\mathrm{T}$ (SNP5) and 2722G $>\mathrm{C}$ (SNP12) in at least one of the two study groups.

In conclusion, CARD15 mutations, which are important in Crohn's disease and Blau syndrome, play no major role in sarcoidosis in this study population. However, these mutations could be of limited importance, especially in patients without a family history of sarcoidosis.

Eur Respir J 2003; 22: 748-754.
*Institute of Human Genetics, University of Lübeck, Lübeck, ${ }^{\#}$ Dept of General Internal Medicine, Christian-Albrechts-Universtität Kiel, Kiel, and Dept of Pneumology, Medical University Hospital Freiburg, Freiburg, Germany.

Correspondence: M. Schürmann, Institute of Human Genetics, University of Lübeck, Lübeck, Germany.

Fax: 494515004187

E-mail: schuerma@medinf.mu-luebeck.de

Keywords: Blau syndrome, CARD15 gene mutations, Crohn's disease, family study, sarcoidosis

Received: May 162002

Accepted after revision: July 92003

This study was funded by the German Federal Ministry of Research and Education (BMBF) through the German National Genome Research Network (NGFN) programme and its clinical network "Inflammation and Environment" (Grant \#: 01 GS 01160101 - 01 GS 0121), the German Human Genome Project and the European Commission's 5th Framework programme.
Sarcoidosis, Blau syndrome and Crohn's disease are complex disorders. They are all associated with chronic inflammation and show a remarkable overlap in the spectrum of organs involved and in their immunopathophysiology. Both sarcoidosis and Crohn's disease are characterised by an enhanced immune response to a yet unknown target, with monocyte and T-cell activation and granuloma formation in the affected organs [1-3]. The primary sites of sarcoidosis and Crohn's disease manifestation are the lungs and intestines, respectively, where close contacts to exogenous agents take place. Both disorders are most prevalent in young adults with a slight preponderance of females. The incidence rate of sarcoidosis is $\sim 10-20$ in 100,000 [4] and of Crohn's disease is $\sim 15-50$ in 100,000 in European populations [5], with considerable regional differences. Both disorders cluster in close relatives in approximately five out of 100 affected families [5-9]. Coincidence of Crohn's disease and sarcoidosis in a family or even in one individual patient is more common than expected by chance alone [9]. Skin, eyes, joints and other organs are commonly concomitantly affected in sarcoidosis and this can also be seen in Crohn's disease.

Blau syndrome, a rare inherited disorder, is characterised by granulomatous arthritis, uveitis and erythema [10]. It is caused by a single dominant gene and starts in early childhood. Based on similarities in the clinical presentation, it has been suggested that Blau syndrome is a monogenic early-onset variant of sarcoidosis. The Blau syndrome gene has been mapped close to the centromere of chromosome 16 by linkage analysis in an extended multi-case pedigree [11]. Susceptibility to Crohn's disease has been mapped to a locus (IBD1) in the same chromosomal region by several studies of large cohorts of siblings suffering from Crohn's disease [12-14].

It has been demonstrated, in several independent studies, that distinct alleles of the CARD15 (also referred to as NOD2) gene are associated with Crohn's disease [15-17], with considerable genotype/phenotype correlation and a genotype relative risk of up to 23.4 for homozygotes or compound heterozygotes [18-20]. The CARD15 gene resides on the long arm of chromosome 16 close to the centromere (16q12) and the CARD15 protein is composed of two caspase recruitment domains (CARD), a nucleotide oligomerisation domain (NOD) and a stretch of leucine rich repeats (LRR). An important function of CARD15 may be the activation of the nuclear regulatory factor (NF)- $\mathrm{B}$ pathway in response to stimulation with microbial lipopolysaccharides. High $N F-\kappa B$ activity is a consistent feature in Crohn's disease [21] and sarcoidosis [22]. It has been suggested that single nucleotide polymorphisms (SNPs) in or close to the LRR, which are strongly associated with Crohn's disease, may alter NF- $\kappa \mathrm{B}$ activation in response to lipopolysaccharides. Alternatively, mutations in the CARD15 gene may influence the regulation of immune cell apoptosis [23]. Subsequently, mutations of the same gene, CARD15, have been detected in families with Blau syndrome $[24,25]$. However, while the most significant Crohn's 
disease-associated CARD15 mutations are located in or close to the LRR region, Blau syndrome mutations were found to cluster in the NOD, thus suggesting that alterations of different segments of the gene cause or contribute to different granulomatous disorders.

The similarities and overlaps of sarcoidosis, Blau syndrome and Crohn's disease warrant an exploration of CARD15 gene variations in sarcoidosis. To this end, a sample of 138 families with more than one patient suffering from sarcoidosis and 127 unrelated patients together with their parents were studied for mutations in CARD15.

\section{Subjects and methods}

Three study populations were analysed: 1) families with two or more members suffering from sarcoidosis (sarcoidosis families); 2) unrelated sarcoidosis patients without a family history of sarcoidosis, together with their parents (sarcoidosis trios); and 3) a sample of healthy control individuals who matched the patients of the sarcoidosis families with respect to sex ratio and average age.

The complete families panel comprised 302 patients (135 males, 167 females; average age at inclusion (mean \pm SD) $51 \pm 13.1 \mathrm{yrs}$, average age at diagnosis $35 \pm 11.3 \mathrm{yrs}$, range $12-73$ yrs) and 153 close relatives without sarcoidosis (64 males, 89 females). Patients of the trios sample were enrolled if diagnosis was proven by histology (with the exception of two patients with classical Löfgren syndrome) and both parents agreed to participate. The trios sample consisted of 127 patients (German Caucasians; 49 males, 78 females; average age at inclusion $34 \pm 6.8 \mathrm{yrs}$, average age at diagnosis $33 \pm 6.9$ yrs, range $11-51$ yrs) and 254 parents without sarcoidosis. The control group was comprised of 265 healthy German Caucasian blood donors, recruited through the Dept of Transfusion Medicine at the Kiel University Hospital, Kiel, Germany (118 males, 147 females; average age at inclusion $51 \pm 8.2$ yrs).

The sample of sarcoidosis families (138 German families, Caucasians) included 66 families with two or three siblings suffering from sarcoidosis, 41 families with a pair of affected parent and offspring, 19 families with two or more distantly related patients and 12 families (44 patients) with more than two patients and a more complex structure (table 1).

Most patients were contacted by calls for participation via the German patients' organisation, others came from specialised hospitals and interested practitioners. All index patients (first contacts to the families) from sarcoidosis families and the majority of other patients from sarcoidosis families were briefly interviewed by telephone about their sarcoidosis history and family structure. All patients and their physicians were consulted by questionnaire for information concerning the diagnosis and course of disease. Sarcoidosis was confirmed by biopsy in 209 out of 302 cases of the family sample and in 125 out of 127 cases of the trios, and the clinical course was consistent with the diagnosis of sarcoidosis in the remaining patients. All participants gave written informed consent to participate in the study, including genotype analysis.

Using the Taqman ${ }^{\mathrm{TM}}$ system [19], all individuals of the three samples (total 1,101) were genotyped for four Crohn's disease-associated CARD15 polymorphisms: SNP5 (802C $>$ T, P268S, nomenclature according to [18]); SNP8 (2104C $>$ T, R702W); SNP12 (2722G>C, G908R); and SNP13 (3020insC, $1007 \mathrm{fs})$. The rarer alleles of these biallelic polymorphisms are named allele 1 in the following text. Allele and genotype frequencies were compared for all SNP in cases from the families and trios samples and controls with Chi-squared statistics. Only one randomly selected patient per family
Table 1. - Structure of 138 families with two or more patients suffering from sarcoidosis

\begin{tabular}{lcccc}
\hline Relationship of patients & $\begin{array}{c}\text { Families } \\
\mathrm{n}\end{array}$ & $\begin{array}{c}\text { Patients } \\
\mathrm{n}\end{array}$ & $\begin{array}{c}\text { Males } \\
\mathrm{n}\end{array}$ & $\begin{array}{c}\text { Females } \\
\mathrm{n}\end{array}$ \\
\hline Affected siblings & & & & \\
$\quad$ Sister/sister & 22 & 44 & & 44 \\
$\quad$ Sister/brother & 27 & 54 & 27 & 27 \\
$\quad$ Brother/brother & 11 & 22 & 22 & \\
$\quad$ Three siblings & 6 & 18 & 9 & 9 \\
Affected parent/offspring & 18 & 36 & & 36 \\
$\quad$ Mother/daughter & 8 & 16 & 8 & 8 \\
$\quad$ Mother/son & 5 & 10 & 5 & 5 \\
$\quad$ Father/daughter & 10 & 20 & 20 & \\
$\quad$ Father/son & 19 & 38 & 21 & 17 \\
Affected 2 and 3 ${ }^{\circ}$ relatives & & & & \\
Complex families & 7 & 21 & 9 & 12 \\
$\quad$ Three patients & 3 & 12 & 9 & 3 \\
$\quad$ Four patients & 1 & 5 & 1 & 4 \\
$\quad$ Five patients & 1 & 6 & 4 & 2 \\
$\quad$ Six patients & 138 & 302 & 135 & 167 \\
Total & & & & \\
\hline
\end{tabular}

of the sarcoidosis families sample was included in this case-control analysis to exclude related patients and to avoid over-representation of specific alleles from multi-case families. In addition, family-based analyses were performed: transmission disequilibrium tests in the sarcoidosis families and in the trios; and nonparametric linkage analysis in the sarcoidosis families only, using TRANSMIT [26] and GENEHUNTER [27]. Furthermore, on the basis of haplotype information from a previous genome-wide linkage study of highly polymorphic deoxyribonucleic acid (DNA) marker (microsatellite) genotypes in 63 families [28], 39 sib-pairs or trios with at least one parental chromosome 16 in common were identified. One patient from each of these sib-pairs or trios, together with eight patients who represent seven complex families with more than two affected siblings who have no parental chromosome 16 in common, were chosen for DNA sequence analysis of exon 4 (exon designation according to [18]) of CARD15. Overlapping polymerase chain reaction (PCR) products were generated using two primer pairs (5'-TCCCTTCAGTTATGTCAGCGTC-3' and 5'-CAGAGAAGCCCTTGAGGTTGA-3'; and 5'-AAACCACTCTCTGTGCGGACTC-3' and 5'-AGAACGCGGCAAAGAAGCA-3'), thus covering 1,175 basepairs of exon 4 , including the sites of missense mutations found in Blau syndrome [24, 25]. Sequence analysis was performed using BigDye $^{\mathrm{TM}}$ chemistry (Aplera Inc., Foster City, CA, USA) according to the manufacturer's protocol. In brief, $2 \mathrm{ng}$ of patient DNA were amplified by PCR. PCR products were purified using SAP/Exol digestion and then used in the sequencing reaction. Sequences were read on ABI 3700 automated sequencers (Aplera Inc.).

\section{Results}

\section{Single nucleotide polymorphism analyses}

A total of 1,011 individuals from three study samples of sarcoidosis families, sarcoidosis trios and controls were genotyped for four Crohn's disease-associated CARD15 polymorphisms, SNP5, SNP8, SNP12 and SNP13. The observed genotype proportions within the groups of controls and cases from sarcoidosis families and sarcoidosis trios were all in agreement with expectations of the Hardy-Weinberg law. The controls in this study had previously been part of a 
North European population investigated in a study of CARD15 mutation haplotype structure, which revealed strong linkage disequilibrium between SNP5, SNP8, SNP12 and SNP13 [29]. $\mathrm{D}^{\prime}$ values of pair-wise linkage disequilibrium calculations from the present study ranged from 0.87 (SNP8-SNP13) to 1 (SNP8-SNP12; SNP12-SNP13) and are consistent with previous findings.

Three different types of data analysis were performed: casecontrol comparisons, genetic linkage analyses and family-based association studies. Comparisons of SNP allele frequencies in 265 controls and 265 cases (138 cases from 138 sarcoidosis families, one patient at random chosen from each family, and 127 cases from 127 sarcoidosis trios) revealed significantly $(\mathrm{p}<0.05)$ increased frequencies of SNP5 allele 1 and SNP12 allele 1 in cases. Cases from sarcoidosis families and from sarcoidosis trios contributed to this effect in SNP12, but only cases from sarcoidosis families showed a significant increase of SNP5 allele 1. According to allele frequencies, SNP5 and SNP12 genotypes were different in cases and controls, with a significant difference of SNP12 genotypes in sarcoidosis trios and combined cases from trios and families. All case-control comparisons are listed in tables 2 and 3.

Genetic linkage analysis, performed as an investigation of affected siblings from the sarcoidosis families sample, showed no significant over-representation of any of the studied SNP alleles on chromosomes shared by affected siblings [27]. The best nonparametric linkage score of $0.41(\mathrm{p}=0.33)$ was gained for SNP12, but the information content of the biallelic polymorphisms was low (best information content of 0.23 for SNP5). If haplotypes of the four adjacent SNP were considered in multi-point linkage analysis, the nonparametric linkage score was $0.16(\mathrm{p}=0.43$; information content 0.3$)$ over the entire chromosomal locus under investigation. In six out of 10 families with more than two affected siblings, the rare CARD15 mutations, SNP8, SNP12 or SNP13, were found in at least one of the patients (10 out of 33 patients). However, in five of these six families the CARD15 mutations did not co-segregate with sarcoidosis. In fact, the only patient homozygous for the rare SNP13 allele had two affected siblings negative for the analysed CARD15 mutations (fig. 1).

Family-based association studies were performed as transmission disequilibrium tests, checking for deviation from random transmission of SNP alleles from heterozygote parents to affected offspring. One-hundred and nine complete trios could be extracted from the sarcoidosis families sample, in addition to 127 trios of the sarcoidosis trios set. There was no transmission disequilibrium in the sarcoidosis families sample, neither for single SNPs nor for haplotypes of up to all four SNPs of the study. By contrast, transmission of SNP5 allele were distorted in the sarcoidosis trios sample, with significantly increased transmission of allele 1 and decreased transmission of allele 2 from heterozygote parents to affected

Table 2. - CARD15 allele frequencies in patients suffering from sarcoidosis and in healthy controls

\begin{tabular}{|c|c|c|c|c|}
\hline CARD15 alleles & Controls & Sarcoidosis families & Sarcoidosis trios & Sarcoidosis families +trios \\
\hline Subjects $n$ & 265 & 138 & 127 & 265 \\
\hline SNP5 1 & $144(27.2)$ & $97(35.1)$ & $80(31.5)$ & $177(33.4)$ \\
\hline SNP5 2 & $386(72.8)$ & 179 (64.9) & $174(68.5)$ & 353 (66.6) \\
\hline p-value & & 0.019 & 0.21 & 0.027 \\
\hline SNP8 1 & $25(4.7)$ & $15(5.4)$ & $19(7.5)$ & $34(6.4)$ \\
\hline SNP8 2 & $505(95.3)$ & $261(94.6)$ & $235(92.5)$ & $496(93.6)$ \\
\hline p-value & & 0.66 & 0.12 & 0.23 \\
\hline SNP12 1 & $4(0.8)$ & $8(2.9)$ & $8(3.2)$ & $16(3.0)$ \\
\hline SNP12 2 & $526(99.2)$ & $268(97.1)$ & $246(96.8)$ & $514(97.0)$ \\
\hline p-value & & 0.017 & 0.011 & 0.007 \\
\hline SNP13 1 & 19 (3.6) & $11(4.0)$ & $8(3.2)$ & 19 (3.6) \\
\hline SNP13 2 & $511(96.4)$ & $265(96.0)$ & $246(96.8)$ & $511(96.4)$ \\
\hline p-value & & 0.78 & 0.75 & 0.99 \\
\hline
\end{tabular}

Single nucleotide polymorphism (SNP) designation according to [18]. The rarer allele of each SNP is named allele 1.

Table 3.-CARD15 genotype frequencies in patients suffering from sarcoidosis and in healthy controls

\begin{tabular}{|c|c|c|c|c|}
\hline CARD15 genotypes & Controls & Sarcoidosis families & Sarcoidosis trios & Sarcoidosis families + trios \\
\hline Subjects $n$ & 265 & 138 & 127 & 265 \\
\hline SNP5 1/1 & $18(6.8)$ & $17(12.3)$ & $11(8.7)$ & $28(10.6)$ \\
\hline SNP5 $1 / 2$ & $108(40.7)$ & $63(45.7)$ & $58(45.7)$ & $121(45.7)$ \\
\hline SNP5 $2 / 2$ & $139(52.5)$ & $58(42)$ & $58(45.7)$ & $116(43.8)$ \\
\hline p-value & & 0.06 & 0.43 & 0.083 \\
\hline SNP8 $1 / 1$ & 0 & 0 & $1(0.8)$ & $1(0.4)$ \\
\hline SNP8 $1 / 2$ & $25(9.4)$ & $15(10.9)$ & $17(13.4)$ & $32(12.1)$ \\
\hline SNP8 $2 / 2$ & $240(90.6)$ & $123(89.1)$ & $109(85.8)$ & $232(87.5)$ \\
\hline p-value & & 0.65 & 0.17 & 0.37 \\
\hline SNP12 $1 / 1$ & 0 & $1(0.7)$ & 0 & $1(0.4)$ \\
\hline SNP12 $1 / 2$ & $4(1.5)$ & $6(4.3)$ & $8(6.3)$ & $14(5.3)$ \\
\hline SNP12 $2 / 2$ & $261(98.5)$ & $131(95.0)$ & $119(93.7)$ & $250(94.3)$ \\
\hline p-value & & 0.08 & 0.01 & 0.034 \\
\hline SNP13 $1 / 1$ & 0 & 0 & 0 & 0 \\
\hline SNP13 $1 / 2$ & $19(7.2)$ & $11(8.0)$ & $8(6.3)$ & $19(7.2)$ \\
\hline SNP13 2/2 & $246(92.8)$ & $127(92.0)$ & $119(93.7)$ & $246(92.8)$ \\
\hline p-value & & 0.77 & 0.75 & 0.99 \\
\hline
\end{tabular}

Single nucleotide polymorphism (SNP) designation according to [18]. The rarer allele of each SNP is named allele 1. 


\begin{tabular}{|c|c|c|c|c|c|c|}
\hline \multirow[b]{2}{*}{ D16S769 } & & \multicolumn{2}{|c|}{1} & \multicolumn{2}{|c|}{2} & \\
\hline & & 4 & 4 & 4 & 4 & \\
\hline D16S753 & & 7 & 4 & 4 & 2 & \\
\hline D16S411 & & 5 & 2 & 4 & 2 & \\
\hline CARD15/SNP13 & & 2 & 1 & 2 & 1 & \\
\hline D16S3396 & & 6 & 6 & 2 & 5 & \\
\hline D16S419 & & 11 & 11 & 10 & 6 & \\
\hline \multirow[t]{2}{*}{ D16S3252 } & & 4 & 2 & 2 & 2 & \\
\hline & & & \multicolumn{2}{|c|}{4} & & 5 \\
\hline D16S769 & 4 & 4 & 4 & 4 & 4 & 4 \\
\hline D16S753 & 7 & 4 & 7 & 4 & 4 & 2 \\
\hline D16S411 & 5 & 4 & 5 & 4 & 2 & 2 \\
\hline CARD15/SNP13 & 2 & 2 & 2 & 2 & 1 & 1 \\
\hline D16S3396 & 6 & 2 & 6 & 2 & 6 & 5 \\
\hline D16S419 & 11 & 10 & 11 & 10 & 11 & 6 \\
\hline D16S3253 & 4 & 2 & 4 & 2 & 2 & 2 \\
\hline
\end{tabular}

Fig. 1.-Extended haplotype analysis in a family with three siblings suffering from sarcoidosis $(\boldsymbol{Q}, \mathbf{\square})$, showing discordant segregation of CARD15 single nucleotide polymorphism (SNP)13 alleles and sarcoidosis (D16S***: polymorphic deoxyribonucleic acid loci flanking the CARD15 gene on chromosome 16, genotypes from a previous genome-wide linkage analysis [28]).

offspring. Accordingly, there was a significantly decreased transmission of haplotypes, including SNP5 allele 2. Results of transmission disequilibrium tests are compiled in table 4.

In order to study a possible relation to the age of diagnosis, the patients from sarcoidosis families and trios, respectively, were divided into groups of individuals with diagnosis of sarcoidosis before or at the age of 32 yrs (72 out of 138 patients from the families and 56 patients from the trios sample) or later (66 patients from the families and 71 patients from the trios). Pair-wise comparisons of these subgroups revealed no significant differences of SNP allele or genotype frequencies. In addition, the patient with the younger age at diagnosis was extracted from each of the 138 sarcoidosis families (138 individuals, average age at diagnosis $30 \pm 8.2$ yrs (12-58 range)), as was the patient with the higher age at diagnosis (138 individuals, average age at diagnosis $41 \pm 11.6$ yrs (22-73 range)). Again, no significant differences of SNP allele or genotype frequencies were detected. Transmission disequilibrium tests using the age-related subgroups showed that the preferential transmission of the rarer alleles of SNP5, SNP8 and SNP12 in trios was mainly due to the contribution of trios with patients diagnosed at $\geqslant 33$ yrs. In this subsample, the relation of SNP5 allele 1 transmission to nontransmission was $35 / 20(\mathrm{p}=0.04)$, compared to $25 / 19(\mathrm{p}=0.37)$ in the group of patients with earlier onset of sarcoidosis. There were five trios with patients diagnosed at $\geqslant 33 \mathrm{yrs}$ informative for transmission of SNP13 alleles. In all of these five trios, the rare allele 1 was transmitted to the patient.

\section{Sequence analysis of the nucleotide oligomerisation domain}

In 39 patients (from 39 families) known to be identical by descent with their affected sibling(s) for at least one parental chromosome 16, none of the reported Blau syndrome mutations and no new mutations were detected in exon 4. The same was found in eight patients representing seven complex families with more than two patients who have no parental chromosome 16 in common. Sequence analysis of these 47 patients revealed that the frequencies of the known alleles of the polymorphic sites SNP5, SNP6 and SNP7 of exon 4 were not different from controls (data not shown).

\section{Discussion}

Sarcoidosis and Crohn's disease are multifactorial disorders in which disease is caused by an inherited susceptibility and an additive influence of exogenous agents [1-3]. In contrast, the effect of a single dominant gene is obvious in Blau syndrome, as it directly leads to Mendelian inheritance of the disorder, although as yet unknown factors considerably modify the expression of the disorder between and within affected families $[10,11]$. The clear mode of monogenic inheritance enabled the unequivocal localisation of the Blau syndrome gene to the centromeric region of chromosome 16

Table 4.-Results of transmission disequilibrium tests based on 109 complete trios extracted from the sarcoidosis families sample and on 127 trios of the sarcoidosis trios set

\begin{tabular}{|c|c|c|c|c|c|c|c|c|c|}
\hline & \multicolumn{3}{|c|}{ Families } & \multicolumn{3}{|c|}{ Trios } & \multicolumn{3}{|c|}{ Families and trios } \\
\hline & Transm. & Untransm. & p-value & Transm. & Untransm. & p-value & Transm. & Untransm. & p-value \\
\hline SNP5 1 & 31 & 33 & 0.8 & 60 & 39 & 0.034 & 91 & 72 & 0.13 \\
\hline SNP8 1 & 8 & 8 & & 18 & 10 & 0.13 & 26 & 18 & 0.23 \\
\hline SNP12 1 & 5 & 5 & & 8 & 3 & 0.13 & 13 & 8 & 0.28 \\
\hline SNP13 1 & 8 & 7 & 0.8 & 8 & 8 & & 16 & 15 & 0.86 \\
\hline Haplotype: 1122 & 8 & 7 & 0.8 & 14 & 7 & 0.13 & 22 & 14 & 0.18 \\
\hline Haplotype: 1212 & 5 & 4 & 0.74 & 6 & 2 & 0.16 & 11 & 6 & 0.23 \\
\hline Haplotype: 1221 & 3 & 6 & 0.32 & 8 & 6 & 0.59 & 11 & 12 & 0.83 \\
\hline Haplotype: 1222 & 18 & 19 & 0.87 & 31 & 23 & 0.28 & 49 & 42 & 0.46 \\
\hline Haplotype: 2122 & 0 & 0 & & 1 & 3 & 0.32 & 1 & 3 & 0.32 \\
\hline Haplotype: 2212 & 0 & 0 & & 0 & 0 & & 0 & 0 & \\
\hline Haplotype: 2221 & 4 & 1 & 0.18 & 0 & 0 & & 4 & 1 & 0.18 \\
\hline Haplotype: 2222 & 25 & 26 & 0.89 & 33 & 52 & 0.039 & 58 & 78 & 0.086 \\
\hline
\end{tabular}

Only the results of the rarer allele 1 of individual single nucleotide polymorphisms (SNP) are listed. For allele 2 of the biallelic polymorphisms, the number of transmitted (Transm.) and untransmitted (Untransm.) alleles is inverse, with identical p-values. Haplotypes are formed by alleles of SNP5SNP8-SNP12-SNP13. 
by linkage analysis of only one extended family [11]. Large numbers of affected families and repeated genome-wide linkage studies were necessary to confirm the existence of a gene involved in Crohn's disease in the same chromosomal region [12-14] and this strategy finally led to the identification of CARD15 gene mutations as major contributors to Crohn's disease susceptibility [15-17]. In sarcoidosis, genome-wide linkage information is so far limited to one report [28], showing a main peak at the major histocompatability complex region on the short arm of chromosome 6 , in agreement with results of numerous association studies [30-32]. However, this peak cannot fully explain the increased familial recurrence risk found in different studies in European populations $[6,7,9]$ and in the USA [8]. Rather, the contribution and cooperation of several genetic disease factors in the development of sarcoidosis is likely.

In addition to a systematic genome-wide search by linkage analysis in affected siblings, the analysis of candidate genes is regarded as an important approach. However, heterogeneity of sarcoidosis, representing a variable influence of a different mixture of genetic factors and different ethnic backgrounds, may conflict results of such studies and could be the reason for contrasting reports on associations between sarcoidosis and polymorphisms in candidate genes [31]. The search continues for promising candidate genes that could contribute to the susceptibility to sarcoidosis. From the current concept of sarcoidosis, this search focuses on genes that are involved in the signalling pathway from an inhaled microbial trigger to a balanced cellular immune response [33].

Increased levels of NF- $\mathrm{NB}$ play an important role in the development and maintenance of inflammation in Crohn's disease [21]. Since the function of the CARD15 protein includes the regulation of $\mathrm{NF}-\kappa \mathrm{B}$ levels, alterations in the CARD15 gene in Crohn's disease patients seem to clarify an important link in the pathway from a presumed exogenous agent to granulomatous inflammation of the intestine and other organs. Increased levels of NF- $\mathrm{NB}$ have also been found in sarcoidosis [22] and significant familial clustering of sarcoidosis and Crohn's disease has been reported [9], hence suggesting homologous mechanisms in the pathophysiology of both disorders.

Blau syndrome is a rare condition with a limited number of reports in the literature. To date there are no detailed studies on the pathophysiology of Blau syndrome and the first report on mutations was based on four unrelated families [24]. All cases from these families carried a mutation in the NOD of the CARD15 gene. In three families the same amino acid of the NOD, arginine at amino acid position 334 of the protein, was substituted (R334Q and R334W) and in two of them an identical point mutation $(1001 \mathrm{G}>\mathrm{C}, \mathrm{R} 334 \mathrm{Q})$ was found. However, this was probably due to independent mutations, as indicated by different flanking marker haplotypes of the mutated chromosomes. More recently, these results were confirmed by demonstration of CARD 15 mutations in five out of 10 unrelated families [25], with R334Q and R334W in three and two families, respectively. Therefore, Blau syndrome mutations appear to cluster strongly within the NOD, and mutations of the NOD and LRR seem to be linked to clearly different phenotypic consequences within the spectrum of granulomatous disorders, leading to the development of Blau syndrome or to a significant increase in the risk of Crohn's disease, respectively.

Taking into account the considerable overlap of clinical presentation in sarcoidosis, Blau syndrome and Crohn's disease, and the coincidence of sarcoidosis and Crohn's disease in patients and families, CARD15, now linked to the pathogenesis of Blau syndrome and Crohn's disease, could be considered an excellent candidate gene of sarcoidosis susceptibility.
There is preliminary information concerning the Blau syndrome gene region in sarcoidosis. RYBICKI et al. [34] have analysed a panel of 33 African-American nuclear families with 67 siblings suffering from sarcoidosis for co-segregation of the disease, with eight highly polymorphic DNA markers from the centromeric region of chromosome 16, and found no evidence of linkage. However, considerable differences of sarcoidosis presentation in African-American and Caucasians, with respect to incidence rates, pattern of organ involvement and familial recurrence risk $[1,8]$, point to heterogeneity of sarcoidosis between ethnic groups, and results from the this study may not be valid in Caucasians. A panel of 63 Caucasian families, a subgroup of the study population presented here, has been analysed previously in a genome-wide scan to localise predisposing genes in sarcoidosis by linkage to a set of microsatellite DNA polymorphisms [28]. The DNA locus D16S3396 of the marker panel used is located close to the CARD15 gene, with a distance of approximately $0.6 \times 10^{6}$ basepairs between both sites. There was no significant evidence $(\mathrm{p}=0.21)$ that siblings suffering from sarcoidosis tend to be identical by descent for this region of chromosome 16, as would be expected in the case of a major contribution of CARD15 to the aetiology of sarcoidosis.

However, the linkage data do not exclude the significance of CARD15 mutations in a subgroup of patients. If mutations of the CARD15 gene contribute to sarcoidosis in only a portion of families, these mutations presumably should reside on a chromosome 16 that affected siblings have concordantly inherited from one of their parents. In the present study the authors analysed the sequence of the CARD15 gene region altered in Blau syndrome and excluded mutations other than known polymorphic sites in a selected panel of patients who were identical by descent for one or two parental chromosomes 16s. This result from sarcoidosis families cannot exclude a minor influence of rare NOD sequence variants in sarcoidosis, but it makes a major contribution, at least in Caucasian patients, unlikely, since a major gene effect would be linked to familial occurrence of sarcoidosis and should be soon detectable in such a panel of selected patients as studied here.

In order to uncover a minor contribution of more common CARD15 DNA variations to the aetiology of sarcoidosis, extended panels of sarcoidosis families, sarcoidosis trios and controls were genotyped for four SNPs associated with increased risk of Crohn's disease. With the exception of SNP13 in the sarcoidosis families, the rarer alleles of the four SNPs tended to be more frequent in cases than in controls and the difference was significant for SNP5 in sarcoidosis families and for SNP12 in sarcoidosis families and in sarcoidosis trios. The allele frequencies of SNP5, SNP8 and SNP12 in the controls are in agreement with published frequencies in controls in a Europe-wide study of Crohn's disease [18], although the SNP13 allele 1 frequency in the current study is considerably higher (3.6 versus 2\%). Conversely, a German control sample included in another study of Crohn's disease showed an even higher SNP13 allele 1 frequency of 5.3\% [16]. From genotyping of the extended group of available German control individuals who were excluded because of young age from the matched control group of this study, it is know that the SNP12 allele 1 frequency in the matched control group is relatively low. Therefore, significant differences to sarcoidosis cases could be attributed, in part, to this control grouprelated phenomenon.

The limitations of case-control comparisons in association studies of candidate gene polymorphisms in complex disorders have been discussed and family-based strategies like the transmission disequilibrium test (TDT) have been developed to overcome control group-related problems [35]. In addition, further information, such as haplotype segregation, 
can be extracted from family-based settings. There is strong linkage disequilibrium between the SNPs in this study, as documented by the haplotype proportions of the TDT analysis. As listed in table 4, the rare alleles of SNP8, SNP12 and SNP13 occur almost exclusively in combination with SNP5 allele 1. In the trios sample, all haplotypes with the rare allele of at least one the four SNPs are preferentially transmitted from heterozygote parents to affected offspring, even though this deviation does not reach significance. However, there is significant nontransmission of the complementary haplotype composed of the four more common alleles. Interestingly, transmission of alleles and haplotypes is almost perfectly balanced in the sarcoidosis families sample and this fact could point to heterogeneity in the genetic aetiology of sarcoidosis with respect to CARD15 gene variation.

In summary, the results of association, transmission and sequence analysis in this study rule out a major role of CARD15 mutations that are of significance in Crohn's disease and Blau syndrome in the pathogenesis of sarcoidosis in Caucasians. This finding demonstrates genetic heterogeneity of granulomatous inflammatory disorders, although clinical phenotypes and immunopathophysiology show significant overlap. A minor contribution of CARD15 variants cannot be excluded, though evidence is weak, with the exception of SNP12 allele 1 frequencies in case-control comparison. Replication of the investigation is desirable and appears from the transmission disequilibrium test results to be more promising in sarcoidosis trios without a family history of the disease.

\footnotetext{
Acknowledgements. The authors would like to thank the patients and their families who participated in this study. The patients' organisation Deutsche Sarkoidose-Vereinigung contributed essentially to the recruitment of families. K. Wurm, K. Kögler and T. Dieringer (Höchenschwand), U. Costabel (Essen), D. Kirsten (Großhansdorf), G. Liebetrau (Lostau), W. Vorderstrasse (Bremen) and many others supported by introducing the authors to additional families.
}

\section{References}

1. Statement on sarcoidosis. Joint Statement of the American Thoracic Society (ATS), the European Respiratory Society (ERS) and the World Association of Sarcoidosis and Other Granulomatous Disorders (WASOG) adopted by the ATS Board of Directors and by the ERS Executive Committee, February 1999. Am J Respir Crit Care Med 1999; 160: 736755.

2. Müller-Quernheim J. Sarcoidosis: immunopathogenetic concepts and their clinical application. Eur Respir J 1998; 12: 716-738.

3. Podolsky DK. Inflammatory bowel disease. $N$ Engl $\mathrm{J} \mathrm{Med}$ 1991; 325: 928-937.

4. Demedts M, Wells AU, Anto JM, et al. Interstitial lung diseases: an epidemiological overview. Eur Respir J 2001; 18: Suppl. 32, 2s-16s.

5. Shivananda S, Lennard Jones J, Logan R, et al. Incidence of inflammatory bowel disease across Europe: is there a difference between north and south? Results of the European Collaborative Study on Inflammatory Bowel Disease (ECIBD). Gut 1996; 39: 690-697.

6. Kirsten D. Sarcoidosis in Germany. Analysis of a questionnaire survey in 1992 of patients of the German Sarcoidosis Group. Pneumologie 1995; 49: 378-382.

7. McGrath DS, Daniil Z, Foley P, et al. Epidemiology of familial sarcoidosis in the UK. Thorax 2000; 55: 751-754.
8. Rybicki BA, Iannuzzi MC, Frederick MM, et al. Familial Aggregation of Sarcoidosis. A case-control etiologic study of sarcoidosis. Am J Respir Crit Care Med 2001; 164: 20852091.

9. Wirnsberger RM, de Vries J, Wouters EF, Drent M. Clinical presentation of sarcoidosis in The Netherlands: An epidemiological study. Neth J Med 1998; 53: 53-60.

10. Blau EB. Familial granulomatous arthritis, iritis, and rash. J Pediatr 1985; 107: 689-693.

11. Tromp G, Kuivaniemi H, Raphael S, et al. Genetic linkage of familial granulomatous inflammatory arthritis, skin rash, and uveitis to chromosome 16. Am J Hum Genet 1996; 59: 1097-1107.

12. Hugot JP, Laurent-Puig P, Gower-Rousseau C, et al. Mapping of a susceptibility locus for Crohn's disease on chromosome 16. Nature 1996; 379: 821-823.

13. Hampe J, Schreiber S, Shaw SH, et al. A genomewide analysis provides evidence for novel linkages in inflammatory bowel disease in a large European cohort. Am J Hum Genet 1999; 64: 808-816.

14. Cavanaugh $\mathbf{J}$ and The IBD International Genetics consortium. International collaboration provides convincing linkage replication in complex disease through analysis of a large pooled data set: Crohn disease and chromosome 16. Am J Hum Genet 2001; 68: 1165-1171.

15. Hugot JP, Chamaillard M, Zouali H, et al. Association of NOD2 leucine-rich repeat variants with susceptibility to Crohn's disease. Nature 2001; 411: 599-603.

16. Ogura Y, Bonen DK, Inohara N, et al. A frameshift mutation in NOD2 associated with susceptibility to Crohn's disease. Nature 2001; 411: 603-606.

17. Hampe J, Cuthbert A, Croucher PJ, et al. Association between insertion mutation in NOD2 gene and Crohn's disease in German and British populations. Lancet 2001; 357: $1925-1928$.

18. Lesage S, Zouali $\mathrm{H}$, Cezard JP, et al. CARD15/NOD2 mutational analysis and genotype-phenotype correlation in 612 patients with inflammatory bowel disease. Am J Hum Genet 2002; 70: 845-857.

19. Cuthbert AP, Fisher SA, Mirza MM, et al. The contribution of NOD2 gene mutations to the risk and site of disease in inflammatory bowel disease. Gastroenterology 2002; 122: $867-874$

20. Hampe J, Grebe J, Nikolaus S, et al. NOD2 genotype and clinical course of Crohn's disease. Lancet 2002; 359: 16611665.

21. Schreiber S, Nikolaus S, Hampe J. Activation of nuclear factor kappa B inflammatory bowel disease. Gut 1998; 42: 477-484.

22. Drent $M$, van den Berg R, Haenen GR, van den Berg $H$, Wouters EF, Bast A. NF-kappaB activation in sarcoidosis. Sarcoidosis Vasc Diffuse Lung Dis 2001; 18: 50-56.

23. Beutler B. Autoimmunity and apoptosis: the Crohn's connection. Immunity 2001; 15: 5-14.

24. Miceli-Richard $\mathrm{C}$, Lesage $\mathrm{S}$, Rybojad M, et al. CARD15 mutations in Blau syndrome. Nat Genet 2001; 29: 19-20.

25. Wang $\mathrm{X}$, Kuivaniemi $\mathrm{H}$, Bonavita $\mathrm{G}$, et al. CARD15 mutations in familial granulomatosis syndromes: a study of the original Blau syndrome kindred and other families with large-vessel arteritis and cranial neuropathy. Arthritis Rheum 2002; 46: 3041-3045.

26. Clayton D. A generalization of the transmission/disequilibrium test for uncertain-haplotype transmission. Am J Hum Genet 1999; 65: 1170-1177.

27. Kruglyak L, Daly MJ, Reeve-Daly MP, Lander ES. Parametric and nonparametric linkage analysis: a unified multipoint approach. Am J Hum Genet 1996; 58: 13471363.

28. Schürmann M, Reichel P, Müller-Myhsok B, Schlaak M, Müller-Quernheim J, Schwinger E. Results from a genome-wide search for predisposing genes in sarcoidosis. Am J Respir Crit Care Med 2001; 164: 840-846. 
29. Croucher PJ, Mascheretti S, Hampe J, et al. Haplotype structure and association to Crohn's disease of CARD15 motations in two ettinically divergent populations. Eur J Hum Genet 2003; 11: 6-16.

30. Schürmann M, Lympany PA, Reichel $\mathrm{P}$, et al. Familial sarcoidosis is linked to the major histocompatibility complex region. Am J Respir Crit Care Med 2000; 162: 861-864.

31. Luisetti M, Beretta A, Casali L. Genetic aspects in sarcoidosis. Eur Respir J 2000; 16: 768-780.

32. Sato H, Grutters JC, Pantelidis P, et al. HLA-DQB1*0201: a marker for good prognosis in British and Dutch patients with sarcoidosis. Am J Respir Cell Mol Biol 2002; 27: 406412.

33. McGrath DS, Goh N, Foley PJ, du Bois RM. Sarcoidosis: genes and microbes - soil or seed? Sarcoidosis Vasc Diffuse Lung Dis 2001; 18: 149-164.

34. Rybicki BA, Maliarik MJ, Bock $\mathrm{CH}$, et al. The Blau syndrome gene is not a major risk factor for sarcoidosis. Sarcoidosis Vasc Diffuse Lung Dis 1999; 16: 203-208.

35. Spielman RS, Ewens WJ. The TDT and other family-based tests for linkage disequilibrium and association. Am J Hum Genet 1996; 59: 983-989. 